\title{
Prevalence of Malaria Infection and ABO Human Blood Group in Dar-es-Salaam, Tanzania
}

\author{
John Nwangwu1 ${ }^{*}$, Ivan Roland², David Mbelwa ${ }^{3}$, Cassian Magori ${ }^{3}$, Marian Evans ${ }^{1}$, Cynthia Price ${ }^{4}$ \\ ${ }^{1}$ Department of Public Health, Southern Connecticut State University, New Haven, CT, USA \\ ${ }^{2}$ Department of Physiology, Hubert Kairuki Memorial University, Dar-es-Salaam, Tanzania \\ ${ }^{3}$ Department of Anatomy, Hubert Kairuki Memorial University, Dar-es-Salaam, Tanzania \\ ${ }^{4}$ Department of Emergency Medicine, Hartford Hospital, Hartford, CT, USA \\ Email: *Nwangwuj1@southernct.edu
}

How to cite this paper: Nwangwu, J. Roland, I., Mbelwa, D., Magori, C., Evans, M. and Price, C. (2017) Prevalence of Malaria Infection and $\mathrm{ABO}$ Human Blood Group in Dar-es-Salaam, Tanzania. $A d$ vances in Infectious Diseases, 7, 153-157. https://doi.org/10.4236/aid.2017.74016

Received: June 20, 2017

Accepted: December 1, 2017

Published: December 4, 2017

Copyright $\odot 2017$ by authors and Scientific Research Publishing Inc. This work is licensed under the Creative Commons Attribution International License (CC BY 4.0).

http://creativecommons.org/licenses/by/4.0/

\begin{abstract}
The main objective of this study was to determine the relationship between occurrence of malaria infection with that of human blood group in Dar-es-Salaam Tanzania. The study was based on data collected randomly from the patients who were screened for malaria of all age groups for a period of three months at Mission Mikocheni Hospital. During this time period, a total of 430 blood samples were collected. From the study findings, it is clear that more than $50 \%$ of the patients tested were positive for malaria. Further analysis revealed that about $50 \%$ of malaria positive patients were type "O" blood group.
\end{abstract}

\section{Keywords}

Prevalence, Malaria Infection, ABO Human Blood Group

\section{Introduction}

Malaria is a protozoal parasitic disease which is widely distributed in tropical countries. It is caused by minute parasitic protozoa of the genus plasmodium with high morbidity and mortality [1]. There are four species of plasmodium which affect humans, they are: Plasmodium falciparum; Plasmodium malariae; Plasmodium vivax, Plasmodium ovale [2]. Malaria parasites have been with us since the dawn of time. They probably originated in Africa and accompanied human migration to the different parts of the modern world [2]. The malaria parasite has two hosts, human and insect. The insect host (mosquito) is the definitive host, in which the parasite develops and multiplies by sexual and asexual 
cycles. The plasmodium parasites are highly specific with humans as the only vertebrate host and anopheles mosquitoes as the vectors [3].

The distribution of malaria parasites depends on the habits of its vectors (i.e., Anopheles mosquitoes) which are influenced by many factors/stimuli like odors, carbon dioxide, chemicals, body temperature, humidity, age of the host and blood type, which are all believed to attract them to their human host [4]-[11]. Existing knowledge suggest that what attracts mosquitoes to humans is the need for food since their food is human blood [3] [12]. However what makes some individuals more attractive to the insects than other individuals is part of this investigation.

Most humans belong to the $\mathrm{ABO}$ blood group system which consists of four major blood groups and eight different blood types. $\mathrm{A}, \mathrm{B}, \mathrm{AB}$, and $\mathrm{O}$ are the various combinations and can be negative or positive depending on the presence or absence of antigens or antibodies which gives us the eight types. It has long been known that a persons' susceptibility to malarial infection differs with blood groups [13]. Some have suggested that some species of anopheles mosquito have preferential feeding to certain blood groups, while blood groups may confer protection from severe malarial infection. This may give survival or selection preference to certain blood groups like group O [13] [14], as well as contributing to the distribution of group O persons globally [14].

Since we know that insects are the vectors for many human diseases, by understanding the mechanism of the attraction of these insects to human, it will contribute to the control of insect-human contact and thus to the control of many diseases transmitted by insects.

\section{Aim and Objectives}

1) To observe and record the distribution of malaria infection among a sample of patients from the Dar-es-Salaam region of Tanzania.

2) To quantify the association of the clinical presentation of malaria infection to the various blood groups in the Dar-es-Salaam region of Tanzania.

\section{Materials and Methods}

This was a cross-sectional investigation conducted at the Mission Mikocheni Teaching Hospital of Hubert Kairuki Memorial University in Dar-es-Salaam Tanzania following approval. The blinded data was collected over a period of 90 days in 2016. A total sample of 430 randomly selected patients of all ages and sexes from the Dar-es-Salaam region were included. The patients presented to the hospital with a variety of illnesses, and were subjected to routine laboratory examination for malaria at the Mission Mikocheni Teaching Hospital. Laboratory analysis was performed to determine two main factors: 1) Malaria sero-status "positive" or "Negative"; and 2) ABO blood typing was performed only among the patients that were seropositive for malaria infection. Malaria infection was confirmed by light microscopy and Quantitative Buffy Coat (QBC). Hospital identification cards were verified to confirm that they were from the 
Dar-es-Salaam region. Odds ratio (OR) was calculated using a two by two frequency table.

\section{Results}

Out of the 430 patient samples, $282(65.8 \%)$ were found to be seropositive for malaria infection and $148(34.2 \%)$ were found to be seronegative for malaria infection (see Table 1). The blood group analysis showed that 68.8 (24.1\%) were "A" blood group, 58.9 (20.9\%) were "B" blood group, 14.9 (5.3\%) were "AB" blood group and 139 (49.3\%) were "O" blood group (see Table 2). Descriptive statistics were not available. Odds ratio (ORs) with 95\% confidence intervals (CIs) were reported (see Table 3). A weak association was shown between $\mathrm{O}$ blood groups and seropositivity for malaria (see Table 3). Other recent studies show similar association observations (see Table 4).

\section{Discussion}

The results of this investigation show that the prevalence of malaria infection in the Dar-es-Salaam region is 65.8 percent. The investigation also revealed that the maximum number of participants that tested "positive" for malaria infections were found in blood group type $\mathrm{O}$ which was 49.6 percent followed by blood group type $A$ at 24.1 percent, then blood group type B was 21 percent and blood group type $\mathrm{AB}$ had the least infected blood group type of 5.3 percent. These results are similar to the results found in the investigation done by Tewodros et al.,

Table 1. Distribution of positive malaria infection, $\mathrm{N}=430$.

\begin{tabular}{ccc}
\hline Status & Number $(\mathbf{n})$ & Percentage (\%) \\
\hline Positive & 282 & 65.8 \\
Negative & 148 & 34.2 \\
Total & 430 & 100 \\
\hline
\end{tabular}

Table 2. Malaria infection and blood groups, $\mathrm{N}=282$.

\begin{tabular}{ccc}
\hline Blood group & \# of Malaria infections & Percentages (\%) \\
\hline Group A & 68.8 & 24.1 \\
Group B & 58.9 & 20.9 \\
Group AB & 14.9 & 5.3 \\
Group O & 139 & 49.3 \\
Total & 282 & 100 \\
\hline
\end{tabular}

Table 3. Measures of association between malaria infection and blood group.

\begin{tabular}{ccc}
\hline Blood group & Odds ratio & $95 \%$ C.I. \\
\hline A & 0.84 & $0.71-1.00$ \\
B & 0.91 & $0.64-1.31$ \\
AB & 0.81 & $0.55-1.16$ \\
O & 1.28 & $0.43-3.87$ \\
\hline
\end{tabular}


Table 4. Prevalence of malaria infection and blood groups in recent studies.

\begin{tabular}{ccccc}
\hline Recent authors & A (\%) & B (\%) & AB (\%) & O (\%) \\
\hline Nwangwu & 24.1 & 21 & 5.3 & 49.6 \\
Singh (2015) & 16.1 & 40.9 & 13.0 & 25.9 \\
Hussain (2014) & 32.3 & 35.3 & 24.2 & 17.7 \\
Gayatri (2013) & 16.1 & 40.9 & 8.8 & 34.2 \\
Olajevwo (2013) & 34.6 & 23.1 & 3.9 & 38.4 \\
Deepa (2011) & 22.0 & 42.0 & 1.0 & 35.0 \\
Tewodros (2011) & 23.5 & 21.9 & 3.3 & 51.3 \\
Sing et al. (1995) & 14.3 & 11.1 & 0 & 13.9 \\
\hline
\end{tabular}

2011 (see Table 4) [7]. In this study, they found blood group type O with the highest number of malarial infection of 51.3 percent followed by blood group type A with 23.5 percent, then blood group type B with 21.9 percent, and blood group type $\mathrm{AB}$ with the least number of malarial infections at 3.3 percent [7]. Our results are re-assuring given that two previous investigations were done in East Africa, which is in the same region of the world, Tewodros et al. investigation was done in Ethiopia and this present investigation was done in Tanzania. It is also worthy to note that six out of eight investigations presented in Table 4 have the same pattern of results with blood group type $O$ having the highest number of malarial infections, followed by type $A$, then type $B$, and the least number of infections being in type $\mathrm{AB}$ blood group.

\section{Conclusion}

The data from this investigation shows that the prevalence of malaria in the Dar-es-Salaam region and Tanzania for the period 2016-2017 is $65.8 \%$. It could be suggested that perhaps the prevalence is high partly because there are more blood group type "O" in the area. The data in this investigation also shows that the blood group typing of $\mathrm{ABO}$ in human beings may suggest differences in susceptibility to malaria infections. The data also confirms that persons having blood group type "O" are more prevalent with malaria infections in areas endemic to malaria.

\section{References}

[1] Pathirana, S., Alies, H., Bandara, S., Phon, K., Perera, M., Wickremasinghe, A. and Handunnetti, S. (2005) ABO Blood-Group Types and Protection against Severe Plasmodium falciparum Malaria. Annals of Tropical Medical Parasitology, 99, 119-124. https://doi.org/10.1179/136485905X19946

[2] Coetzee, M., Craig, M. and Sueur, D. (2000) Distribution of African Malaria Mosquitoes Belonging to the Anopheles gambiae Complex. Parasitology Today, 16, 74-77. https://doi.org/10.1016/S0169-4758(99)01563-X

[3] Constantini, C., Sagnon, N., Tore, A. and Cozuzzi, M. (1999) Mosquitoes Behavioural Aspects of Vector Human Interactions in Anopheles gambiae Complex. Parasitologia, 41, 209-217. 
[4] Singh, N., Shukla, M., Uniyal, V. and Sharma, V. (1995) ABO Blood Groups among Malaria Cases from District Mandla, Madhya Pradesh, India. Journal of Malariology, 32, 59-73.

[5] Coluzzi, M. and Bradley, D. (1999) The Malaria Challenge. Parasitologia, 41, 361-376.

[6] Deepa, V., Alwar, K. and Cecil, R. (2014) ABO Blood Groups and Malaria Related Clinical Outcome. Journal of Vector Borne Diseases, 48, 7-11.

[7] Tewodros, Z., Abraham, D. and Bethanu, E. (2011) Association of ABO Blood Group and Plasmodium falciparum Malria in Dore Bafeno Area, Southern Ethiopia. Asia Pacific Journal of Biomedical Science, 1, 289-294. https://doi.org/10.1016/S2221-1691(11)60045-2

[8] Gayathri, B., Harendra, K., Gomathi, N., Jeevan, S. and Reethesh, R. (2013) Relationship between ABO Blood Groups and Malaria with Clinical Outcome in Rural Area of South India. Global Journal of medical Public Health, 2, 1-7.

[9] Otajevwo, F. (2013) Prevalence of Malaria Parasitemia and Its Association with ABO Blood Grouping among Students of Igbinedion University Okada, Nigeria. British Medical Journal, 3, 1161-1177.

[10] Hussain, S., Idachaba, S. and Idoko, T. (2014) Susceptibility of humans of the ABO Blood Groups to Plasmodium falciparum Infection among Patients Attending Ahmadu Bello University Clinic (Sickbay), Samaru-Zaria, Kaduna State, Nigeria. School Journal of Applied Medical Sciences, 2, 1305-1309.

[11] Singh, G., Urhekar, A. and Singh, R. (2015) A Study on Correlation of Malaria Infection with A, B, O, RH Blood Group System. Journal of Parasitology and Vector Biology, 7, 67-73.

[12] Maibach, H., Skinner, W., Strauss, W. and Khan, A. (1966) Factors that Attract and Repel Mosquitoes in Human Skin. Journal of the American Medical Association, 196, 173-176. https://doi.org/10.1001/jama.1966.03100160113032

[13] Gupta, M. and Chowdhuri, A. (1980) Relationship between ABO Blood Groups and Malaria. Bulletin of the World Health Organization, 58, 913-915.

[14] Karolinska Institutet (2015) How Blood Group O Protects against Malaria. Science Daily. https://www.sciencedaily.com/releases/2015/03/150309124113.htm 